\title{
Die vorflutigen Chaldäerfürsten des Annianos.
}

Bekanntlich haben Panodoros und Annianos die Sage des Enochbuches vom Abfall der Egregoren benutzt, um für die mythischen Dynastien der Ägypter und Babylonier in der vorflutigen Periode Raum zu gewinnen. Die Rechnung des Annianos, welcher nur Nachtreter des Panodoros ist, giebt Michael der Syrer (S. 6 ff. der Ausgabe von Jerusalem, vgl. S. 23 der Übersetzung von Langlois) in wünschenswerter Deutlichkeit wieder. Da ich bei der Behandlung von Panodoros' chaldäischer Geschichte in meinem Africanus II S. $198 \mathrm{ff}$. dieses wichtige Bruchstück aus Annianos' Chronographie unbenutzt gelassen habe, gebe ich hier den Text in wörtlicher Übersetzung:

„Im 40sten Jahre Jareds vollendeten sich 1000 Jahre. Und in diesem Jahre stiegen die Söhne Seths, da sie sich langweilten, vom Berge Ahermon herunter und verfielen auf den Wunsch nach Weibern. Und sie waren zweihundert an der Zahl. Und zu derselben Zeit setzten sie einen König ein, dessen Name Samarios war. Und darüber berichtet Enanos ${ }^{1}$ ), dås, weil die Söhne Seths vom Berge hinabgestiegen und vom engelgleichen Leben (abfielen), ihre Brüder ergrimmten und ihnen keine Weiber gaben. Und sie gingen hin und nahmen von den Töchtern Kains, welche ihnen gefielen. Und von ihnen stammten die frechen verderblichen Riesen und Mörder. Und bis zu dieser Zeit bestand die Furcht Gottes und Friede, und da dies aufgehoben war, beherrschte sie Aufruhr und Krieg und Mord. Und dies hörten die Söhne Seths, welche im zweiten Klima (Klimn), was Abteilung bedeutet, lebten, und sie setzten sich selbst einen König ein im Lande der Chaldäer (K'a $\lambda$ dêaçik'), den Aגoros. Dieser erfand die Astronomie, die Zahl der Tierkreisbilder, der Planeten und ihrer Umläufe. Und von ihm empfing (diese Wissenschaft) den Namen Chaldaismus (K'addêut yn). Und dieser war König 98 Jahre.

Und nach diesem war König sein Sohn Elp'aros 29 Jahre und 255 Tage.

Und nach diesem war König Almion ein Chaldäer aus der Stadt P'udivilon 128 Jahre und 80 Tage.

Vierter König war Amanon ein Chaldäer 118 Jahre und 130 Tage.

Fünfter König war Amakaros ein Chaldäer 177 Jahre und 197 Tage.

1) Langlois S. 23 giebt nach seinen Handschriften den Zusatz: 'qui emprunta ce récit au livre d'Enoch', welcher in der Ausgabe von Jerusalem fehlt. 
Sechster König war Dinos der Hirte ein Chaldäer 98 Jahre und 230 Tage.

S. 7. Siebenter König war Evturink os ein Chaldäer 175 Jahre und 195 Tage.

Achter König war Mampios ein Chaldäer aus der Stadt Anark on 195 Jahre und 230 Tage.

Neunter König ,war Otiartos aus der Stadt Anarké on 78 Jahre und 330 Tage.

Zehnter König war K'sisot'ros, sein Sohn, 177 Jahre und 195 Tage.

Das ergiebt zusammen 1183 Jahre und 205 Tage. Und in seinen Tagen war die Flut nach der chaldäischen Schrift. Zu der Zeit der Könige 1183 (Variante: 1180) (addiere man) die Zeit ohne Königtum 1052 und es ergeben sich im ganzen bis zur Flut 2242 Jahre gemäfs . der von Gott eingegebenen Schrift."

Ich stelle nun im folgenden die Zahlen des Berossos denen des Michael sowohl nach der Ausgabe von Jerusalem, wie nach der von Venedig gegenüber; die wahren Zahlen des Annianos ergeben sich dann von selbst.

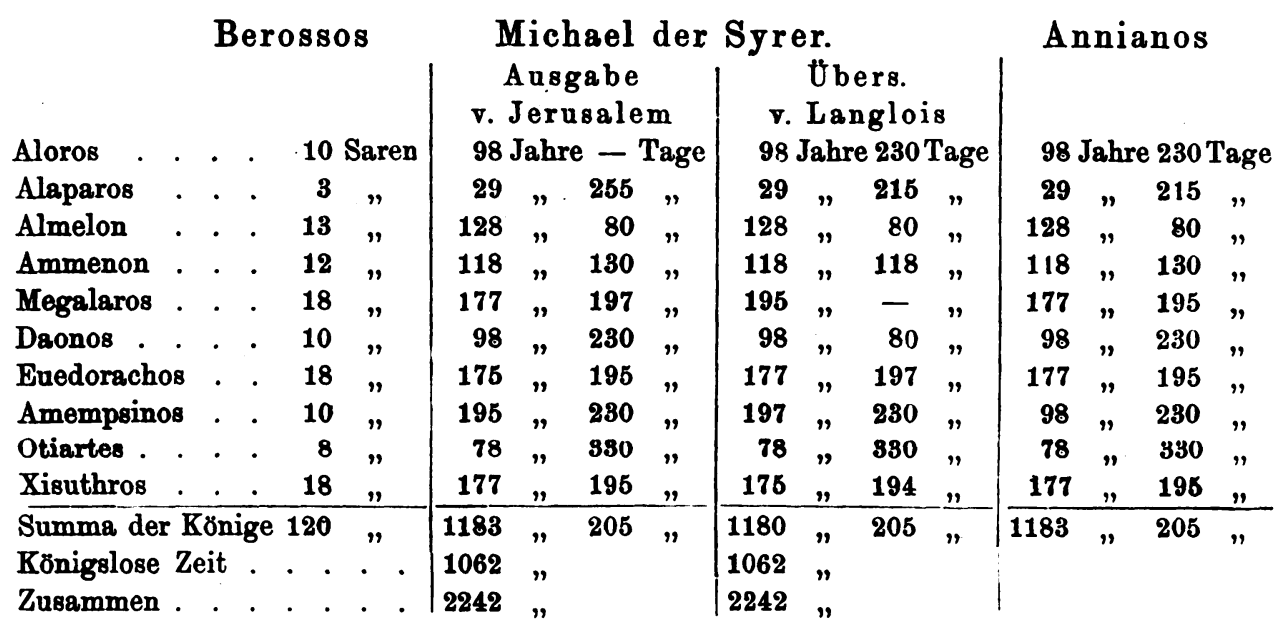

Den Schlüssel zur Reduktion des Panodoros und seines Nachtreters

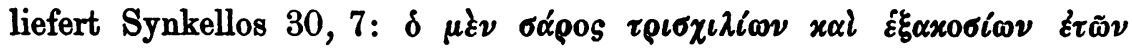

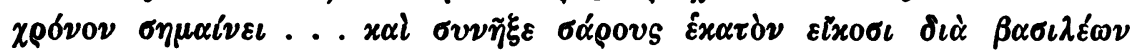

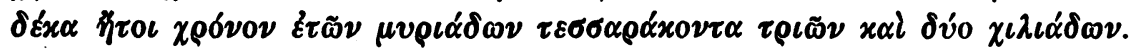

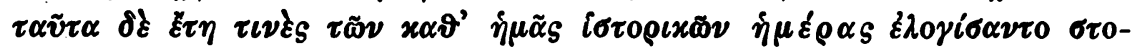

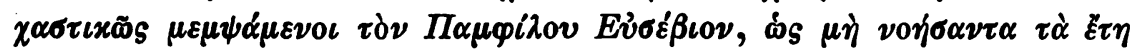

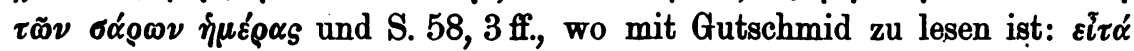

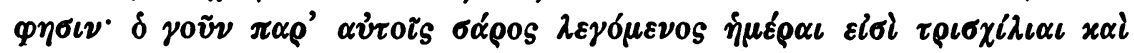

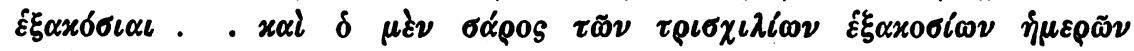




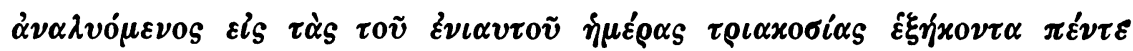

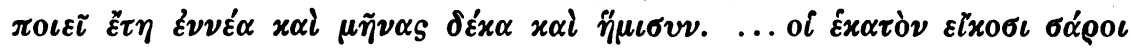

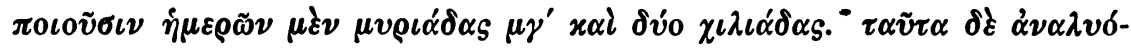

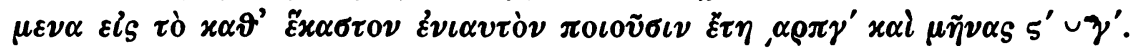

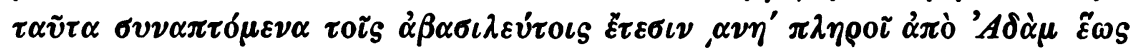

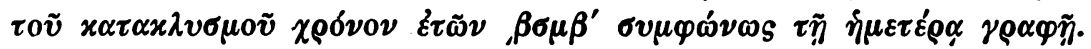

Danach ist Annianos' Rechnung ganz klar; die 120 Saren sind 432000 Tagjahre, welche nun in Sonnenjahre von 365 Tagen verwandelt und auf die einzelnen Königsregierungen reduziert werden. Die Fehler der beiden Rezensionen Michaels sind unbedeutend und erklären sich vielfach paläographisch, sehr leicht aus den armenischen Zahlzeichen. Was zuerst die Ausgabe von Jerusalem betrifft, so ist bei Aloros die Tagzahl ausgefallen. Bei Alaparos ist die Tagzahl 255: $\boldsymbol{S} \&$ statt $\$ \&$; Megalaros hat 197 Tage: $x_{q} t$ statt $x_{q} t$; Euedorachos 175 Jahre: $x_{s t}$ statt $x_{55}$; Amempsinos hat $x_{q} t$, auch beim Venetianer $x_{q} 5$ Jahre statt qૃ (98); der Fehler, weil in beiden Rezensionen vorhanden, ist also möglicherweise sehr alt. Langlois' Ubersetzung hat beim vierten König 118 Tage, Duplikat aus der Jahreszahl und beim fünften 195 Jahre; die Tageszahl hat die Jahreszahl verdrängt; Euedorachos hat 197 Tage $\left(\gamma_{\eta t}\right.$ statt $\left.\gamma_{z}^{\prime} r\right)$. Endlich Xisuthros hat $\gamma<\xi$ statt $\gamma<5$ Jahre. Wie man sieht, sind das alles nur ganz leichte Schreibfehler, die sich bei der vollkommenen Klarheit der ganzen Rechnung mit Sicherheit bessern

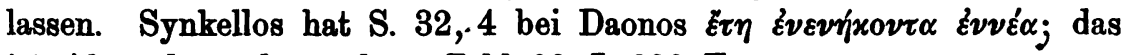
ist Abrundung der wahren Zahl 98 J. 230 T.

Die Summe ergiebt, wie die Jerusalemer Ausgabe ganz richtig hat, 1183 Jahre 205 Tage; die Lesart 1180 Jahre ist lediglich Verschlimmbesserung eines aufmerksamen Diorthoten, welcher die Summe 2242 erreichen wollte. Allein der Fehler steckt vielmehr in der königslosen Jahrzahl 1062. Auch hier bietet das Richtige Synkellos 29, 15: $\tau \tilde{\boldsymbol{\sigma}}$

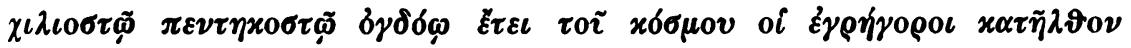

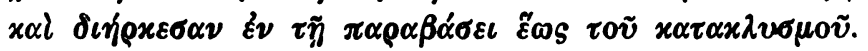

Michael S. 10 und Synkellos 39, 20 setzen den $x \alpha \tau \alpha x \lambda v \sigma \mu o ̀ s$ auf den 27. Iar $=20$. Mai 2242; wenn wir von diesem Datum die 1183 Jahre 205 Tage abziehen, gelangen wir in den 28. Oktober des Weltjahrs 1058, was aufs schönste mit der Angabe des Synkellos übereinstimmt.

So hat sich hier durch Vermittelung der Syrer und Armenier ein wenn auch nicht gerade sehr wertvolles, aber zur Kenntnis. der Methode jener Mäinner interessantes Stück christlich alexandrinischer Chroncgraphenarbeit erhalten.

Jena.

H. Gelzer. 